\title{
Article
}

Arq Neuropsiquiatr 2011;69(3):446-451

\section{Primary progressive aphasia patients evaluated using diffusion tensor imaging and voxel based volumetry-preliminary results}

\author{
Fábio Pascotto de Oliveira', Jaderson Costa da Costa², \\ Sabine Possa Marroni ${ }^{3}$, Ana Maria Marques da Silva4, \\ Sthefanie Hansen Barreiro ${ }^{5}$, Fábio Kunihiro Maeda ${ }^{6}$, \\ Mirna Wetters Portuguez ${ }^{7}$
}

\begin{abstract}
There are individuals who have a progressive language deficit without presenting cognitive deficits in other areas. One of the diseases related to this presentation is primary progressive aphasia (PPA). Objective: Identify by means of diffusion tensor imaging (DTI) and measurements of cortical volume, brain areas that lead to dysphasia when presenting signs of impaired connectivity or reduced volume. Method: Four patients with PPA were evaluated using DTI, and measurements of cortical volumes in temporal areas. These patients were compared with two normal volunteers. Results: There is a trend to a difference in the number and volume of related fibers between control group and patients with PPA. Comparing cortical volumes in temporal areas between groups yielded a trend to a smaller volume in PPA patients. Conclusion: Patients with PPA have a trend to impairment in cortical and subcortical levels regarding relevant areas.
\end{abstract}

Key words: anomia, primary progressive aphasia, DTI, tractography, arcuate fasciculus, language

Avaliação de pacientes com afasia primária progressiva com imagem tensor de difusão e volumetria baseada em voxels

\section{RESUMO}

Existem indivíduos que apresentam deterioração progressiva da linguagem sem apresentar déficits em outros domínios cognitivos; estes pacientes em geral apresentam afasia primária progressiva (APP). Objetivo: Identificar, pela imagenologia, áreas cerebrais que quando sofrem atrofia, ou quando seu sistema de conexões apresenta alterações, levam a disfasia. Método: Foram avaliados quatro pacientes com APP, utilizando-se ressonância magnética com as técnicas de imagem por tensores da difusão (DTI) e medida do volume cortical em áreas de interesse previamente definidas. Estes pacientes foram comparados com um grupo controle constituído por dois voluntários. Resultados: Redução da média dos volumes dos giros temporais esquerdos no grupo de pacientes, em relação ao grupo controle, e alterações quanto ao volume e número de fibras nos tratos correspondentes. Conclusão: Pacientes com APP têm tendência a apresentar danos tanto ao nível cortical quanto subcortical.

\section{Correspondence}

Fábio Pascotto de Oliveira Av. Fernando Ferrari 1067 97050-801 Santa Maria RS - Brasil

E-mail:fpascotto@hotmail.com

Received 12 February 2010

Received in final form 21 December 2010

Accepted 3 January 2011
Palavras-chave: anomia, afasia primária progressiva, DTI, tratografia, volume cerebral, fascículo arqueado, linguagem.

'Neurologist, HUSM, Santa Maria RS, Brazil; ${ }^{2}$ Professor of Neurology, Director Brain Institute, PUCRS, Porto Alegre RS, Brazil; ${ }^{3}$ Unit of Neuropsychology Hospital São Lucas, PUCRS, Porto Alegre RS, Brazil; "Professor of the School of Physics, PUCRS, Porto Alegre RS, Brazil; ${ }^{5}$ Medical Physicist, NIMed, PUCRS, Porto Alegre RS, Brazil; ${ }^{6}$ Physics Student, NIMed, PUCRS, Porto Alegre RS, Brazil; ${ }^{7}$ Professor of Neurology, PUCRS, Porto Alegre RS, Brazil. 
Language is one of the pillars of human intellect, and it is defined as the ability to communicate. It involves associating sounds and symbols with significant concepts and allow us to describe the external environment and abstract thoughts ${ }^{1}$. Currently, one of the most accepted theories about language suggests it is processed in two pathways the ventral and the dorsal ${ }^{2}$. This model proposes that the ventral pathway, which involves structures in portions of the upper and middle temporal lobe, is involved in signal processing for understanding spoken language. Moreover, the dorsal pathway, which involves structures of the posterior region of the frontal lobe, posterior dorsal temporal lobe, and parietal operculum, is involved in translating acoustic signals to articulatory language representations in the frontal lobe. The latter route would be essential for the development of language and its normal production ${ }^{3}$.

Thus oral communication occur as follows, first the speech would be examined in the auditory cortex bilaterally in the supratemporal plane, and processed in phonological and representative levels involving the middle portion of the posterior superior temporal sulcus bilaterally. Subsequently the system splits towards the dorsal and ventral pathway; the dorsal pathway have dominance in the left hemisphere and the ventral pathway is represented bilaterally and has a weak dominance in the left hemisphere. The anatomical areas involved in these pathways are not known perfectly but the approximate locations are described below.

The dorsal pathway, which has a strong dominance in the left hemisphere involves in its posterior aspect an area near the sylvian fissure in the temporoparietal junction, proposed as a sensory-motor interface. And in its anterior aspect it would involve the anterior part of the insula, the inferior frontal gyrus and also the premotor cortex ${ }^{2}$.

The ventral pathway, which has weak dominance in the left hemisphere being organized bilaterally, involves the middle temporal gyrus and inferior temporal sulcus. The most posterior areas of the ventral pathway would correspond to a lexical interface, which would link the phonological information with semantics ${ }^{3}$.

At the subcortical level it has been proposed to the inferior fronto-occipital fasciculus a semantic function, since it was observed that after intraoperative stimulation semantic paraphasias were noticed, regardless of the point in which this pathway was stimulated ${ }^{4}$.

The arcuate fasciculus is classically linked to language, and it has been demonstrated that stimulation in different parts of this structure generates different expressions including phonemic paraphasias, and disorders that affect the formation of phonological words ${ }^{5}$. Currently it is recognized that the arcuate fasciculus that connects the parietal and temporal regions of the frontal lobe is composed of various subcomponents. Besides the classic arcuate fasciculus connecting Wernicke's area and Broca's area directly, there is an indirect route that runs parallel and lateral to the arcuate fasciculus ${ }^{6}$, and this indirect route connects the frontal lobe with the parietal lobe, and the temporal lobe with the parietal lobe.

Knowing the way that language is processed, we can infer that people with speech disorders present some involvement of the related structures. Those who develop a slow and progressive decline in language function without cognitive deficits in other domains, usually have a disease called primary progressive aphasia (PPA) ${ }^{7}$.

The diagnosis of PPA can now be performed in any patient in which the progressive loss of language constitutes the most salient aspect of the clinical condition, when it is caused by a neurodegenerative disease ${ }^{8}$. The damage to language can be fluent or nonfluent and may or may not interfere with word recognition. Memory for recent events recognition of familiar faces and objects are preserved but when tested orally may seem abnormal. Small changes in personality and behavior can occur, but usually do not interfere with the patient's life. For the diagnosis of PPA the aphasia has to be the most salient deficit and the major cause of impaired daily living activity for approximately two years.

This study aims to determine in patients with PPA which structures in cortical or subcortical levels are involved in language deterioration. For this purpose we performed brain magnetic resonance (MR) examinations; measured cortical volumes and used diffusion tensor imaging (DTI) in areas involved in speech.

\section{METHOD}

Subjects

Four right handed, patients with diagnosis of PPA were included aged 59-82 (mean 69.25). This diagnosis was confirmed by at least two neurologists according to previously published criteria ${ }^{9}$ and neuropsychological tests. Exclusion criteria were patients or controls that had any condition that could simulate PPA, or had a historical of a previous neurological disease.

These patients were compared with two age-matched health volunteers aged 65 .

All subjects gave written informed consent and the study was approved by the Internal Review Board.

\section{Image acquisition}

MR was performed on a $1.5 \mathrm{~T}$ scanner (Siemens Avanto $76 \times 18 \mathrm{HQ}$ ).

DTI was performed using a single shot echo-planar imaging technique using 12 directions; matrix $192 \times 192$; echo time 96 ms; repetition time 9600 ms; b=1000 s/ 
$\mathrm{mm}^{2}$; flip angle 90 ; pixel spacing $1.25 \times 1.25 \times 2.5$; slice thickness $2.5 \mathrm{~mm}$.

The pulse sequences used for the morphometric analyses were two three-dimensional, T1-weighted [magnetization prepared rapid gradient echo (MP-RAGE)] scans, with the following parameters: repetition time, $2400 \mathrm{~ms}$; echo time, $3.61 \mathrm{~ms}$; inversion time, $1000 \mathrm{~ms}$; flip angle, $8^{\circ}$; matrix, 192×192; field of view, 192. Each scan took 7 minutes and 42 seconds.

\section{Image analysis}

MR data were transferred to an off-line computer for further analysis. Tractography was applied to the DTI data to reconstruct white matter tracts by successively following the path of preferred direction of water diffusion when fractional anisotropy (FA) is higher than a selected threshold. Using MedINRIA software version 1.9.0 (http://www-sop.inria.fr/asclepios/software/ MedINRIA) diffusion tensors were calculated from all voxels within the brain and fiber tracts were calculated by connecting adjacent voxels with similar principal eigenvectors, using a threshold FA value of 0.2. Only fibers with lengths of $>10 \mathrm{~mm}$ were included. These parameters are similar to those used by others who applied a fiber assignment by continuous tracking algorithm. Regions of interest were drawn in each brain on axial slices by a single coder. Regions of interest were drawn according published DTI atlas ${ }^{10}$. We investigated bilaterally the arcuate fasciculus, inferior fronto-occiptal fasciculus and the inferior longitudinal fasciculus. Variables obtained for statistical comparison included the fractional anisotropy, the volume of fibers, and the number of fibers in each of the structures analysed.

Cortical volume was studied using IBASPM ${ }^{11}$, which is a fully automated, easy volumetric method (http:// thomaskoenig.ch/lester/ibaspm.htm). Volume measurement with IBASPM software is based on an individual brain atlas masked using an automated anatomical labeling. This procedure consists of the following five steps: [1] Segmentation: The MR image is segmented into gray matter, white matter, and cerebral spinal fluid. [2] Normalization: The MR image is normalized to the MNI space to obtain the spatial transformation matrix; using this transformation matrix the gray matter is transformed to MNI space. [3] Automatic labeling: each normalized individual gray matter voxel is labeled based on an atlas of predefined 116 segmented structures. [4] Atlasing: Individual atlases are created from each subject's MR image. The individual atlases are reversely normalized, and the gray matter image in native space is masked using its individual 116 brain structures. [5] Volume statistic: For all individual atlases, the volumes of 116 predefined brain structures are calculated. When the voxel values of the gray matter image exceed the voxel values of the white matter and CSF images, the voxel is included for volume calculation.

Measurements were obtained in $\mathrm{mm}^{3}$ using this method. Structures studied included the superior temporal gyri, medial temporal gyri, inferior temporal gyri, and the supramarginal gyri ${ }^{12}$.

\section{Statistical analysis}

It was applied the nonparametric Mann-Whitney test for comparing several variables between cases and controls. A nonparametric test was chosen because the sample is small, not allowing us to ensure the assumptions necessary for a parametric test for comparison of means. We used also the nonparametric Wilcoxon test to compare variables measured between the left and right hemisphere. The Wilcoxon test was used in this situation because we are comparing two measurements in the same individuals, a fact that leads us to use a test for related samples.

\section{RESULTS}

A visual comparison between patients and controls demonstrates differences observed mainly in the left arcuate fasciculus, the left fronto-occipital and left inferior

Table 1. $P$ value for the Mann-Whitney* test to compare number of fibers between PPA group and control group.

\begin{tabular}{cccccc}
\hline LAF & LIFF & LFLI & RAF & RIFF & RFLI \\
\hline 0.481 & 0.064 & 0.159 & 0.355 & 0.481 & 0.165 \\
\hline
\end{tabular}

*P values for bilateral test; LAF: left arcuated fascilus; LIFF: left inferior fronto-occiptal fasciculus; LFLI: left inferior longitudinal fasciculus; RAF: right arcuate fasciculus; RIFF: right inferior fronto-occiptal fasciculus; RFLI: right inferior longitudinal faciculus.

Table 2. P value for the Mann- Whitney* test to compare tract volume between PPA group and control group.

\begin{tabular}{cccccc}
\hline LAF & LIFF & LFLI & RAF & RIFF & RFLI \\
\hline 0.064 & 0.064 & 0.165 & 0.643 & 0.165 & 0.165 \\
\hline
\end{tabular}

*P values for bilateral test; LAF: left arcuated fascilus; LIFF: left inferior fronto-occiptal fasciculus; LFLI: left inferior longitudinal fasciculus; RAF: right arcuate fasciculus; RIFF: right inferior fronto-occiptal fasciculus; RFLI: right inferior longitudinal faciculus. 


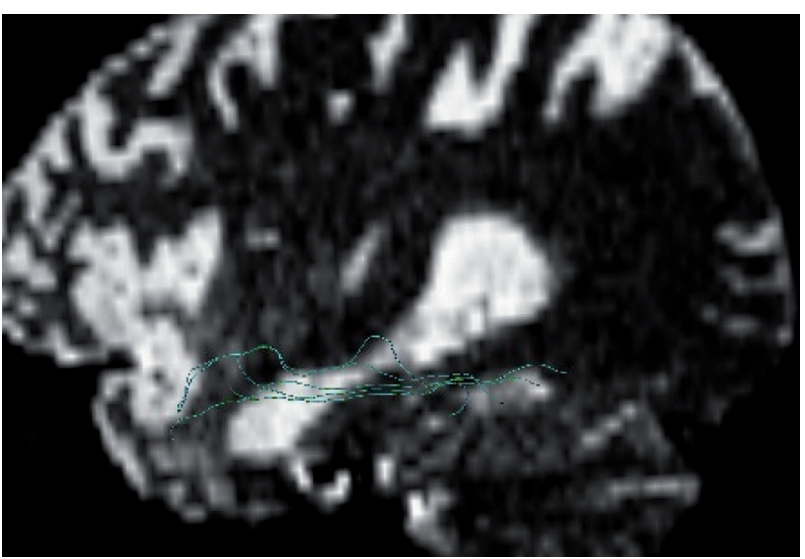

Fig 1. Patient's left inferior longitudinal fasciculus.

longitudinal fasciculus. In Figures 1 and 2 are shown the inferior longitudinal fasciculus in a patient and in a control respectively

When anisotropy was assessed using DTI, we could not find significant difference between the PPA group and the control group when FA was analyzed $(\mathrm{P}=0.24)$. However when compared the number of fibers and volume of fibers there is a trend to a significant difference as shown in Tables 1 and 2.

The cortical volumes for studied areas are shown in Figure 3. It can be seen in this graphic the clear disproportion between groups for all cortical areas except the right supramarginal gyrus (Table 3 ).

The comparison of cortical volumes between areas of both hemispheres for each group is shown in Table 4. In this comparison there is a trend to a difference regarding supramarginal and superior temporal gyri.

\section{DISCUSSION}

Fiber tracts serve as barriers to free water diffusion in the brain. This barrier is the reason for the high anisotropy of white matter when compared to gray matter, in which the diffusion of water is almost isotropic ${ }^{13}$. There are many ways of measuring anisotropy, but one

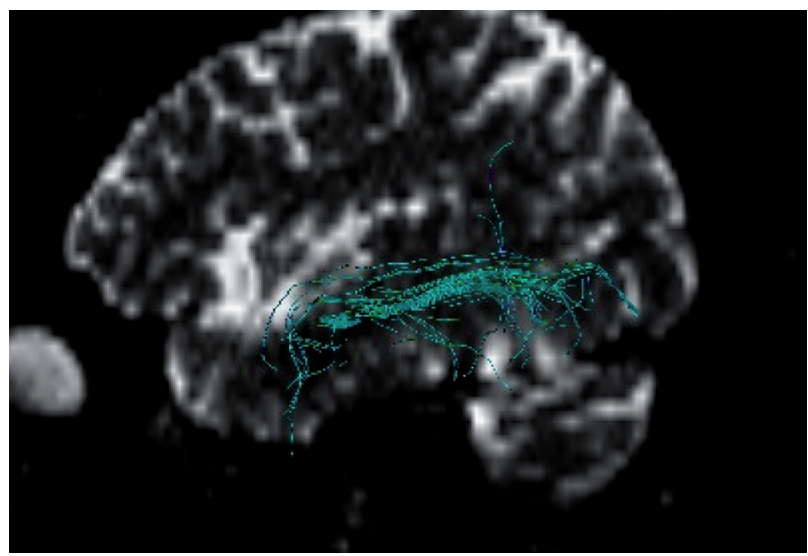

Fig 2. Control left inferior longitudinal fasciculus.

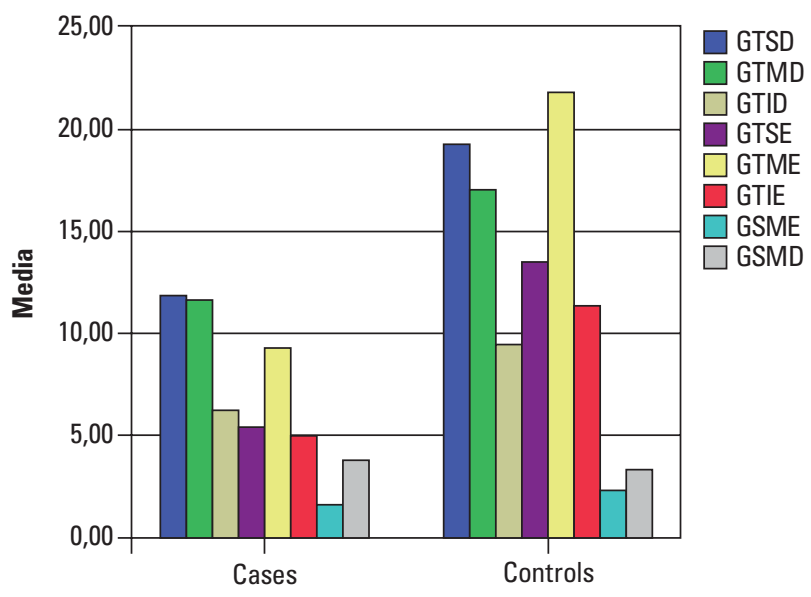

GTSD: right superior temporal gyrus; GTMD: right medium temporal gyrus; GTID: right inferior temporal gyrus; GTSE: left superior temporal gyrus; GTME: left medium temporal gyrus; GTIE: left inferior temporal gyrus; GSME: left supramarginal gyrus; GSMD: right supramarginal gyrus.

Fig 3. Mean cortical volume in $\mathrm{mm}^{3}$ for cases (left side) and controls (right side).

of the most frequently employed is fractional anisotropy, varying from 0 to 1 . If the structures of white matter, axon and myelin are damaged the diffusion of water mol-

Table 3. P values for the Mann-Whitney* test for comparison between cortical volumes between groups.

\begin{tabular}{llllllll}
\hline GTSD & GTMD & GTID & GTSE & GTME & GTIE & GSME & GSMD \\
\hline 0.355 & 0.355 & 0.355 & 0.064 & 0.064 & 0.064 & 0.355 & 0.643 \\
\hline
\end{tabular}

*P values for bilateral test; GTSD: right superior temporal gyrus; GTMD: right medium temporal gyrus; GTID: right inferior temporal gyrus; GTSE: left superior temporal gyrus; GTME: left medium temporal gyrus; GTIE: left inferior temporal gyrus; GSME: left supramarginal gyrus; GSMD: right supramarginal gyrus.

Table 4. $P$ values for the Wilcoxon for comparison of cortical volumes between hemispheres.

\begin{tabular}{cccccc}
\hline Individuals & Number & GTSE $\times$ GTSD & GTME $\times$ GTMD & GTIE $\times$ GTID & GSMD $\times$ GSME \\
\hline Cases & 4 & 0.068 & 0.465 & 0.465 & 0.068 \\
Controls & 2 & 0.180 & 0.180 & 0.180 & 0.180 \\
\hline
\end{tabular}

*P values for bilateral comparison; GTSD: right superior temporal gyrus; GTMD: right medium temporal gyrus; GTID: right inferior temporal gyrus; GTSE: left superior temporal gyrus; GTME: left medium temporal gyrus; GTIE: left inferior temporal gyrus; GSME: left supramarginal gyrus; GSMD: right supramarginal gyrus. 
ecules in these tissues increases, reducing the directionality of the water, thus decreasing the value of the $\mathrm{FA}^{14}$.

Probabilistic tractrography uses the anisotropy of water and tract direction to generate probabilistic maps of connectivity between brain regions and it may draw fibers into the gray matter ${ }^{15}$. The number of fibers in each tract must be understood as a quantitative measure of connectivity between anatomical locations, as determined by the chosen region of interest (ROI) ${ }^{16}$. The loss of fiber loss by axonal degeneration secondary to neuronal injury occur after three months ${ }^{17}$.

One well known limitation of DTI resides in fibber crossings, since there are brain areas in which many fibers with different directions intersect in the same voxel, which typically has dimensions of a few cubic millimeters, but within this area can go up to thousands of axons. In these areas probably this method may have limited use. Another limitation of DTI is that the validation of a tract evidenced by this method is based on prior anatomical knowledge, making it difficult to interpret the absence of a fascicle, or the presence of a tract in place other than the usual ${ }^{18}$. Despite the limitations of DTI, it is the only method for the investigation of brain fibers in vivo ${ }^{14}$.

Evaluation of the white matter integrity using FA has been used in several studies For each disease tracts that may justify patient's condition are studied. In amyotrophic lateral sclerosis research is mainly the pyramidal tract ${ }^{19}$, in Alzheimer's disease the main focus of research is the parahippocampal white matter ${ }^{20}$. This study assessed the arcuate fasciculus bilaterally, the longitudinal fasciculus and inferior fronto-occipital fascicle, because in the existing literature these are subcortical structures most involved in spoken language ${ }^{21}$.

In this study the values of FA are very similar for cases and controls, however when compared the number and volume of fiber tracts, mainly in the left hemisphere between groups it is perceived that patients have a lower number of fibers and a decrease in volume tract volume as shown in Table 1 and 2. Considering what was previously mentioned this could suggest that initially there is a cortical damage with subsequent loss of subcortical structures. It can be inferred that a reduction in the number of fibers can occur in more advanced stages of disease. There is evidence from studies in patients with amyotrophic lateral sclerosis showing that patients with rapid progression of the disease have decreased measures of brain connectivity in the pyramidal tract when compared to controls, and it is suggested that this should occur by degeneration of the motor tract ${ }^{15}$.

This study demonstrated differences of the average volume between patients and controls in the three temporal gyri studied, and also that there is an imbalance of cortical volume in the patient group when comparing cerebral hemispheres in the superior temporal and supramarginal gyri. This is in line with the literature showing a reduction in left perisylvian areas in patients with PPA $^{22}$.

These cortical volumetric changes are consistent with the idea presented above in which there must be cortical cell death, at first, with subsequent changes of subcortical networks.

We would like to highlight the findings of the DTI on the volume and number of fibers of the left inferior fronto-occipital fascicle that was disproportionately affected in the PPA group. This tract when disabled seems to cause semantic paraphasias, which are errors about the meaning of the word. These findings suggest that this tract is related is the semantic stream of the ventral pathway of language ${ }^{21}$.

When compared hemispheres in PPA group, cortical volume of the supramarginal gyri and the superior temporal gyri appear to be reduced in the left hemisphere. This could be explained because the superior temporal gyri are the initial point for both routes of language the ventral and dorsa ${ }^{3,23}$.

The ventral pathway, which processes language has a weak dominance in the left hemisphere, then a lesion in this area would not affect comprehension so importantly. On the other hand, the supramarginal gyrus is a part of the dorsal pathway; this pathway has a strong dominance in the left hemisphere and is involved in the articulation of speech ${ }^{2}$. This explains a more pronounced deficit in speech production.

Our study sought to evaluate the circuit of language in patients with primary progressive aphasia using voxel based measures and diffusion tensor imaging obtained by magnetic resonance imaging of the brain. Although the study sample was small reflecting low frequency of PPA data show that patients with this disorder have a tendency for impairment in cortical and subcortical level.

\section{REFERENCES}

1. Price $C J$. The anatomy of language: contributions from functional neuroimaging. J Anat 2000;197(Pt 3):335-359.

2. Hickok G, Poeppel D. The cortical organization of speech processing. Nat Rev Neurosci 2007;8:393-402.

3. Shalom DB, Poeppel D. Functional anatomic models of language: assembling the pieces. Neuroscientist 2008;14:119-127.

4. Duffau H, Gatignol P, Mandonnet E, Peruzzi P, Tzourio-Mazoyer N, Capelle L. New insights into the anatomo-functional connectivity of the semantic system: a study using cortico-subcortical electrostimulations. Brain 2005;128:797-810.

5. Duffau H, Capelle L, Sichez N, et al. Intraoperative mapping of the subcortical language pathways using direct stimulations: an anatomo-functional study. Brain 2002;125:199-214.

6. Catani M, Jones DK, Ffytche DH. Perisylvian language networks of the human brain. Ann Neurol 2005;57:8-16.

7. Ingles JL, Fisk JD, Passmore M, Darvesh S. Progressive anomia without semantic or phonological impairment. Cortex 2007;43:558-564.

8. Mesulam MM. Primary progressive aphasia: a 25-year retrospective. Alzheimer Dis Assoc Disord 2007;21(Suppl):S8-S11. 
9. Rogalski E, Mesulam M. An update on primary progressive aphasia. Curr Neurol Neurosci Rep 2007;7:388-392.

10. Catani M, Thiebaut de Schotten M. A diffusion tensor imaging tractography atlas for virtual in vivo dissections. Cortex 2008;44:1105-1132.

11. Alemán-Gómez YM-GL, Valdés-Hernandesbn P. IBASPM: Toolbox for automatic parcellation of brain structures CD-Rom in Neurolmage 27 vol. Florence, Italy; 2006.

12. Tae WS, Kim SS, Lee KU, Nam EC, Kim KW. Validation of hippocampal volumes measured using a manual method and two automated methods (FreeSurfer and IBASPM) in chronic major depressive disorder. Neuroradiology 2008;50:569-581.

13. Ciccarelli O, Catani M, Johansen-Berg H, Clark C, Thompson A. Diffusionbased tractography in neurological disorders: concepts, applications, and future developments. Lancet Neurol 2008;7:715-727.

14. Johansen-Berg H, Behrens TE. Just pretty pictures? What diffusion tractography can add in clinical neuroscience. Curr Opin Neurol 2006;19: 379-385.

15. Ciccarelli O, Behrens TE, Altmann DR, et al. Probabilistic diffusion tractography: a potential tool to assess the rate of disease progression in amyotrophic lateral sclerosis. Brain 2006;129:1859-1871.

16. Lindenberg R, Renga V, Zhu LL, Betzler F, Alsop D, Schlaug G. Structural integrity of corticospinal motor fibers predicts motor impairment in chronic stroke. Neurology 2010;74:280-287.

17. Rutgers DR, Fillard P, Paradot G, Tadie M, Lasjaunias P, Ducreux D. Diffusion tensor imaging characteristics of the corpus callosum in mild, moderate, and severe traumatic brain injury. AJNR Am J Neuroradiol 2008;29:1730-1735.

18. Pierpaoli C, Barnett A, Pajevic S, et al. Water diffusion changes in Wallerian degeneration and their dependence on white matter architecture. Neuroimage 2001;13:1174-1185.

19. Aoki S, Iwata NK, Masutani Y, et al. Quantitative evaluation of the pyramidal tract segmented by diffusion tensor tractography: feasibility study in patients with amyotrophic lateral sclerosis. Radiat Med 2005;23:195-199.

20. Rogalski EJ, Murphy CM, deToledo-Morrell L, et al. Changes in parahippocampal white matter integrity in amnestic mild cognitive impairment: a diffusion tensor imaging study. Behav Neurol 2009;21:51-61.

21. Duffau $\mathrm{H}$. The anatomo-functional connectivity of language revisited. New insights provided by electrostimulation and tractography. Neuropsychologia 2008;46:927-934.

22. Gorno-Tempini ML, Dronkers NF, Rankin KP, et al. Cognition and anatomy in three variants of primary progressive aphasia. Ann Neurol 2004;55:335-346.

23. Saur D, Kreher BW, Schnell S, et al. Ventral and dorsal pathways for language. Proc Natl Acad Sci U S A 2008;105:18035-18040. 